
\title{
$\angle S$ Research Square \\ Soft tissue sarcoma in adolescent and young adult patients: A retrospective study using a nationwide bone and soft tissue tumor registry in Japan.
}

\section{Takashi Fukushima}

Department of Orthopaedic Surgery, Jichi Medical University

\section{Koichi Ogura}

Department of Musculoskeletal Oncology, National Cancer Center Hospital

\section{Toru Akiyama ( $\nabla$ toruakiyama827@jichi.ac.jp )}

Department of Orthopaedic Surgery Saitama Medical Center Juchi Medical University https://orcid.org/0000-0001-9493-4768

\section{Katsushi Takeshita}

Department of Orthopaedic Surgery, Jichi Medical University

\section{Akira Kawai}

Department of Musculoskeletal, National Cancer Center Hospital

\section{Research article}

Keywords: Soft tissue sarcoma, Adolescents and young adults, Cancer survival, Japan, Database, MPNST

Posted Date: August 14th, 2020

DOI: https://doi.org/10.21203/rs.2.20871/v2

License: (9) This work is licensed under a Creative Commons Attribution 4.0 International License. Read Full License

Version of Record: A version of this preprint was published at Japanese Journal of Clinical Oncology on April 1st, 2021. See the published version at https://doi.org/10.1093/jjco/hyab044. 


\section{Abstract}

\section{Background}

Compared to young children or older adults, the prognoses of adolescent and young adult (AYA) patients with cancer, i.e., those aged from 15 to 39 years, have not improved. In this study, we focused on soft tissue sarcoma (STS) in AYA patients and aimed to determine whether there is a correlation between the AYA age group and overall poor cancer survival in STS. We further aimed to determine which histologic subtypes are more common in AYA patients and investigate the cause of poor outcomes in this group.

Methods

The medical records of 5853 Japanese patients diagnosed with STS between 2006 and 2013 were accessed from the Bone and Soft Tissue Tumor registry (BSTT). We analyzed and compared the epidemiological features of AYA patients with those of other age groups. The cancer survival rates were calculated using the Kaplan-Meier method. Cox proportional hazards models were used to analyze the prognostic factors for cancer survival. The primary endpoint for prognosis was the occurrence of tumorrelated death.

Results

On multivariate analysis, age was not a prognostic factor for poor cancer survival among these patients. Compared to the same categories in other age groups, the proportions of myxoid/round cell liposarcomas, synovial sarcomas, malignant peripheral nerve sheath tumors (MPNST), primitive neuroectodermal tumor, and rhabdomyosarcoma in AYA patients were the highest, but none of the categories were significantly more prevalent in AYA patients. The cancer survival rates of AYA patients with MPNST were poorer than those of the other age groups; however, AYA age was not a prognostic factor on multivariate analysis in MPNST patients.

\section{Conclusions}

Our study is the first to investigate STS in AYA patients using the nationwide BSTT registry. Our findings demonstrate that AYA age is not a prognostic factor for poor cancer survival among those with STS in Japan.

\section{Background}

Cancer survival rates have significantly improved over time, except among adolescent and young adult (AYA) patients with cancer, i.e., those aged from 15 to 39 years [1]. This has been partly related to the difference in biological behavior, a lower enrollment in clinical trials, and the variability of treatment across settings [2]. AYAs with cancer comprise a unique population and have gained research and media attention in recent years. In 2005, the Joint Progress Review Group of the National Cancer Institute and 
the LiveStrong Foundation in Adolescent and Young Adult Oncology convened to examine the state of science associated with cancer among AYAs [3].

Lymphoma, melanoma, testicular cancer, sarcoma, thyroid cancer, leukemia, and breast cancer are the most common cancers in AYA patients [4]. Of these, sarcomas are the most frequent, accounting for up to $9 \%$ of total malignancies in this population [5]. However, sarcoma is a rare disease with an annual incidence rate of 5.6 per 100,000 individuals in Europe [6]. Furthermore, they have widely diverse pathologies with more than 70 histological subtypes [7], and may develop at any age including childhood, occurring anywhere from the head to the feett, with varying aggressiveness, even within the same histological subtype [8]. It is therefore difficult to obtain the data of sarcoma in AYAs. Moreover, studies focusing on the clinical outcomes of AYAs with sarcoma are scarce.

In 2014, the Bone and Soft Tissue Tumor (BSTT) registry in Japan became available for clinical research. The BSTT is a nationwide organ-specific cancer registry for bone and soft tissue tumors and allows largescale nationwide epidemiological investigations in AYA patients with sarcoma in Japan. We have previously used this database for a retrospective study of bone sarcoma in AYA patients in Japan [9]. In this study, we performed a large-scale nationwide epidemiological investigation of AYA patients with soft tissue sarcoma (STS) in Japan with the aim to determine whether there is a correlation between the AYA age group and overall poor cancer survival for STS and to identify the more common histologic subtypes in this age group. We also investigated the risk factors of the poor outcomes in AYA patients with STS.

\section{Methods}

\section{Data source}

The Japanese Orthopaedic Association (JOA) launched the BSTT registry in the 1950s. It is a nationwide patient data collection system for organ-specific bone and soft tissue tumors. This system includes almost all musculoskeletal malignant tumors in Japan. [8] Detailed data on patients with primary bone and soft tissue tumors (both benign and malignant) and metastatic bone tumors treated at the participating hospitals were collected annually. The survey included basic demographic data of the patient as well as information on the tumor, surgery, and any non-surgical treatment. The follow-up survey was conducted 2, 5, and 10 years after the initial registration. It included information on several outcomes at the time of the latest follow-up.

Although it is similar to the National Cancer Institute's Surveillance, Epidemiology, and End Results Program database, our registry has several advantages. One of these is that treating physicians registered several disease-specific detailed data including histologic findings, treatment modalities, and surgical, functional, and oncologic outcomes. These advantages improved the precision of our registry for detailed epidemiological studies. Use of the data from the BSTT registry for clinical research was approved by the Musculoskeletal Tumor Committee of the JOA in 2014 [9] [10]. This study was approved by the Institutional Review Board of the JOA. 


\section{Data extraction}

A total of 7759 patients with STS listed in the BSTT registry between 2006 and 2013 were identified. Data including the year of registration, demographic characteristics, tumor size, location, grade, histological characteristics, Tumor-Node-Metastasis and Enneking stages, treatment details (surgical vs. nonsurgical), and prognosis at the last follow-up visit (no evidence of disease, alive with disease, death from disease, or death from other causes) were obtained from the database. Liposarcomas were subdivided owing to the variable behavior of the different subtypes. Well-differentiated liposarcomas were excluded because they were considered borderline malignant. Histologic subtypes with larger absolute numbers or a higher ratio in AYA patients were analyzed as an independent histological subtype. The other subtypes were assigned to the high-grade or low-grade sarcoma groups. Patients who were registered less than 1 year from the study enrollment date and those with missing data were excluded. Therefore, the data of 5853 patients with primary STS and a prognosis of over one year were extracted from the database.

\section{Statistical analyses}

The primary endpoint for prognosis was tumor-related death. Cancer survival was defined as the period from the date of diagnosis until tumor-related death and was estimated using the Kaplan-Meier method. Patients without tumor-related deaths or those who died from other causes were censored at their last follow-up visit. The factors associated with survival were analyzed using Cox proportional hazards models. Control variables for multivariate analysis were indicated as "references." These included AYA, female sex, low-grade tumor, tumor size $\leq 5 \mathrm{~cm}$, location of the tumor in the upper extremity, non-surgical treatment, limb salvage after surgical removal of tumor, non-chemotherapy regimen, non-radiation therapy, non-metastatic, and superficial. The alpha level for significance was set at a p value of 0.05 . All statistical analyses were conducted using IBM SPSS version 19.0 (IBM SPSS, Armonk, NY, USA).

\section{Results}

Of the 7759 patients with STS (4309 male and 3450 women) identified, 210 (2.7\%) were aged $\leq 14$ years (children), 1467 (18.9\%) were aged 15-39 years (AYAs), 2771 (35.7\%) were aged 40-64 years (adults), and 3311 (42.7\%) were aged $\geq 65$ years (elderly). The common histologic subtypes were undifferentiated pleomorphic sarcomas, 2030 (26.2\%); myxoid/round cell liposarcomas (MRLS), 956 (12.3\%); synovial sarcomas (SySa), 555 (7.2\%); and malignant peripheral nerve sheath tumors (MPNST), 478 (6.2\%). Meanwhile, the histologic subtypes with a higher ratio in AYA patients were MRLS, 286 (19.5\%); SySa, 259 (17.7\%); MPNST, 130 (8.9\%); primitive neuroectodermal tumor (PNET), 111 (7.6\%); and rhabdomyosarcoma (RMS), 94 (6.4\%).

Table 1 shows the patient characteristics and treatments according to the age groups. The most predominant subtype among the AYA patients was MRLS, 286(19.5\%), followed by SySa, 259 (17.7\%). No other categories demonstrated differences in prevalence in the AYA patient groups when compared to the same categories in other age groups. 
Table 2 shows the overall 5-year cancer survival rates among patients with STS with unadjusted and adjusted hazard ratios (HRs) derived from Cox proportional hazard models. The cancer survival rates of AYA patients with STS were poorer than those of adult age groups. However, they were not poorer than that of the child and the elderly age groups. On multivariate analysis, age was not a prognostic factor for poor cancer survival among AYA patients with STS.

Overall, the prognostic factors for poor cancer survival in patients with STS were age 40-64 years (HR: 1.35, 95\% confidence interval [Cl]: $1.11-1.65, P=0.003$ ), age $\geq 65$ years (HR: $2.28,95 \% \mathrm{Cl}: 1.47-2.34$, $P<0.001$ ), high tumor grade (HR: $3.41,95 \% \mathrm{Cl}: 2.37-4.91, P<0.001$ ), tumor size $>5 \mathrm{~cm}$ and $\leq 10 \mathrm{~cm}$ and $>10$ cm (HR: 1.71 and 2.57, 95\% Cl: 1.38-2.12 and 2.06-3.19, $P<0.001$, respectively), multiple tumor location (HR: 2.32, 95\% Cl: 1.59-3.39, $P=0.014$ ), non-surgical treatment compared with surgery (HR: $0.58,95 \% \mathrm{Cl}$ : 0.49-0.69, P<0.001), amputation (HR: 1.69, 95\% Cl: 1.35-2.11, $P<0.001)$, positive surgical margins (HR: $1.65,95 \% \mathrm{Cl}: 1.27-2.14, P<0.001)$, chemotherapy (HR: $1.59,95 \% \mathrm{Cl}: 1.35-1.87, P<0.001)$, radiation (HR: $1.38,95 \% \mathrm{Cl}: 1.20-1.59, P<0.001)$, the presence of metastases (HR: $4.68,95 \% \mathrm{Cl}: 3.97-5.51, P<0.001)$, and deep tumor location (HR: 1.34, 95\% Cl: 1.07-1.67, $P=0.041$ ). The prevalence of these poor prognostic factors excluding age was not higher among the AYA patients compared with other age groups.

Figure 1 shows the Kaplan-Meier plots for cancer survival rates in patients with STS in general and its subtypes. The cancer survival rates of AYA patients with MPNST were poorer than those of other age groups, whereas those for AYA patients with other histologic subtypes were not significantly different from those of other age groups. Therefore, data from AYA patients with MPNST were analyzed to better understand the factors that caused these poor outcomes.

Table 3 shows the characteristics of MPNST based on age groups. MPNST was more prevalent in male AYA patients, had a larger tumor size, appeared predominantly on the head and neck and in deeper layers, had a higher rate of positive surgical margins, and presented with more metastatic lesions at the first visit in AYA patients than in other age groups.

Table 4 shows the results of univariate and multivariate analyses of the prognostic factors for cancer survival in patients with MPNST. The cancer survival rates of AYA patients with MPNST were poorer than those of other age groups on univariate analyses. Tumor size $>5 \mathrm{~cm}$ and $\leq 10 \mathrm{~cm}$ (HR: $2.20,95 \% \mathrm{Cl}$ : 1.01-4.77, $P=0.046)$, tumor size $>10 \mathrm{~cm}$ (HR: 2.54, 95\% Cl: 1.17-5.50, $P=0.018)$, non-surgical treatment compared with surgery (HR: $0.51,95 \% \mathrm{Cl}: 0.29-0.92, P=0.024$ ), radiation (HR: $1.70,95 \% \mathrm{Cl}: 0.67-2.06$, $P=0.035)$, the presence of metastases (HR: $3.11,95 \% \mathrm{Cl}: 1.76-5.51, P<0.001)$, and deep tumor location (HR: $2.71,95 \% \mathrm{Cl}: 1.23-5.98, P=0.014)$ were prognostic factors for poor cancer survival in patients with MPNST, but who were not AYA.

\section{Discussion}

In this study, we presented the nationwide statistics and outcomes in AYA patients with STS. The cancer survival rates of AYA patients with STS were poorer than those of adult age groups; however, they were not poorer than those of the child and the elderly age groups. Among those with STS, the AYA age range 
was not a prognostic factor for poor cancer survival on multivariate analysis. Although few reports have compared cancer survival in AYA patients and other age groups, it has been suggested that AYA patients with STS have poorer outcomes than those of the child and adult age groups [5]; this differed from our findings. This difference may be related to the functioning of the Japanese health insurance system, in which public medical insurance covers $70-90 \%$ of the treatment costs. This increases to $100 \%$ for people in need. This ensures universal and equal access to medical treatment. Insurance coverage rates are significantly lower in AYA patients in the United States [11], and cancer survivors in this group with no health insurance may not receive cancer-related medical care, as opposed to those with insurance [12].

In this cohort, MRLS was the predominant subtype of STS among AYA followed by SySa, consistent with previous reports $[5,8,13-15]$. We could not compare our results with those of previous reports owing to the differences in age ranges. In addition, those studies also included patients with gastrointestinal stromal tumors and/or Kaposi's sarcomas.

Despite the small number of children included in this study, the survival rates of AYA patients with MPNST were poorer than those of the other age groups. However, multivariate analysis demonstrated that being an AYA was not an independent poor prognostic factor for cancer survival in patients with MPNST. Tumor size $>10 \mathrm{~cm}$, deep tumor location, non-surgical treatment, the presence of metastases, and deep tumor location were poor prognostic factors for cancer survival in patients with MPNST. Compared to other age groups, the characteristics that were more prevalent in AYA patients with MPNST were tumor size $>10 \mathrm{~cm}$, non-surgical treatment, radiation, metastatic lesion(s) at presentation, and deep tumor location. Therefore, certain prognostic factors for MPNST were found particularly more frequently in AYA patients with this tumor. This concordance may be attributed to the poorer survival rates in AYA patients with MPNST than in the other age groups.

One possible reason why the survival rates of AYA patients with MPNST were poorer than those of the other age groups is that a larger part of AYA patients with MPNST may have neurofibromatosis type 1 (NF1). MPNST patients with NF1 have been reported to be significantly younger at the time of MPNST diagnosis than those with sporadic tumors (median age, 26 years vs. 53 years) and have poor outcomes $[16,17]$. Thus, the mean age of MPNST patients with NF1 is within the AYA age group. The 5 -year survival rate of MPNST patients with NF1 ranges from $21 \%$ to $49.7 \%$. Meanwhile, the 5 -year survival rate of MPNST patients with non-NF1 ranges from $42 \%$ to $64.9 \%$ [17-20]. However, our study did not distinguish NF1 patients. Thus, future analysis is required to validate this finding.

Our study has several limitations. First, findings from long-term observation of patients in the past 10 years were not available. Second, although the JOA-certified hospitals treat almost all patients with STS in Japan and the participation of all 89 JOA-certified hospitals in this nationwide registry is compulsory, the participation of other hospitals is voluntary. Therefore, only data from the participating hospitals were analyzed. Third, data on structures (pediatric department, adult department, mixed structures) in which these patients were treated were not included in the database. Fourth, the quality of life (QOL) including social functioning and employment in AYA cancer survivors has become an important health issue in 
recent years; however, the factors related to the QOL were not registered in BSTT [21]. Thus, the QOL of AYA cancer survivors was not analyzed. Fifth, our database does not contain chronological information about chemotherapy and radiotherapy, just contain adjuvant or palliative. Thus, the chemotherapy and/or radiotherapy administered pre- or postoperatively or both were analyzed as merged data.

\section{Conclusion}

In this study, we evaluated the descriptive epidemiology and clinical outcomes of AYA patients with STS using a nationwide and large-scale database. We found that AYA age is not a prognostic factor for poor cancer survival among those with STS in Japan. However, AYA patients with MPNST had a poorer survival compared to other age groups. Our findings will provide useful information for the clinical management of AYA patients with STS. Further studies including larger cohorts with more diverse characteristics are warranted to validate our findings.

\section{Abbreviations}

ASPS, alveolar soft part sarcoma

AYA, adolescent and young adult

BSTT, Bone and Soft Tissue Tumor

CCS, clear cell sarcoma

$\mathrm{Cl}$, confidence interval

HR, hazard ratios

JOA, Japanese Orthopaedic Association

MPNST, malignant peripheral nerve sheath tumors

MRLS, myxoid/round cell liposarcoma

NF1, neurofibromatosis 1

PNET, primitive neuroectodermal tumor

QOL, quality of life

RMS, rhabdomyosarcoma

SySa, synovial sarcoma

\section{Declarations}




\section{Ethics approval and consent to participate}

The research was approved by the Ethics Committee of the Japanese Orthopaedic Association on March 17, 2016. This was a retrospective study performed using data from the Bone and Soft Tissue Tumor registry; the authors were not involved in the collection of this data. Patients were informed that their data would be used for research, and the data were de-identified before addition to the database. Retrieval of the data from this database occurred in an unlinked manner. As the data had been anonymized, the Ethical Guidelines for Epidemiological Research (Ministry of Education, Culture, Sports, Science and Technology, and Ministry of Health, Labour and Welfare of Japan) were not applicable to this study. Based on the Ethical Guidelines on Biomedical Research Involving Human Subjects (Ministry of Education, Culture, Sports, Science and Technology, and Ministry of Health, Labour and Welfare of Japan), clinicoepidemiological studies conducted on medical databases constitute research carried out on pre-existing material and data and do not require any interventions or interactions with patients. For these studies, including this one, written informed consent was not compulsory.

\section{Consent for publication}

Not Applicable.

\section{Availability of data and materials}

The datasets generated or analyzed during the current study are not publicly available as they are anonymized patient data from the Japanese Orthopaedic Association. However, the data are available from the authors upon reasonable request and with permission of the Japanese Orthopaedic Association.

\section{Competing interests}

The authors declare that they have no competing interests.

\section{Funding}

None.

\section{Authors' contributions}

$T F, K O, T A$, and $A K$ contributed to the conception and design of the study. TF, KO, TA, and KT contributed to the analysis of data. All authors contributed to the interpretation of results. TF drafted the article; all authors revised it critically and approved the final version submitted for publication. All authors have read and approved the final manuscript.

\section{Authors' information}

Not Applicable.

\section{Author details}


${ }^{1}$ Department of Orthopaedic Surgery, Saitama Medical Center, Jichi Medical University, 1-847 Amanuma, Omiya-ku, Saitama-shi, Saitama, Japan. ${ }^{2}$ Department of Musculoskeletal Oncology, National Cancer Center Hospital, 5-1-1 Tsukiji, Chuo-ku, Tokyo, Japan. ${ }^{3}$ Department of Orthopaedic Surgery, Jichi Medical University, 3311-1 Yakushiji, Shimotsuke-shi, Tochigi, Japan.

\section{Acknowledgments}

We would like to thank all the patients who volunteered for this study.

\section{References}

1. Tricoli JV, Blair DG, Anders CK, Bleyer WA, Boardman LA, Khan J, et al. Biologic and clinical characteristics of adolescent and young adult cancers: Acute lymphoblastic leukemia, colorectal cancer, breast cancer, melanoma, and sarcoma. Cancer. 2016;122:1017-28.

2. Murphy D, Klosky JL, Reed DR, Termuhlen AM, Shannon SV, Quinn GP. The importance of assessing priorities of reproductive health concerns among adolescent and young adult patients with cancer. Cancer. 2015;121:2529-36.

3. Harlan LC, Lynch CF, Keegan TH, Hamilton AS, Wu XC, Kato I, et al. Recruitment and follow-up of adolescent and young adult cancer survivors: the AYA HOPE Study. J Cancer Surviv 2011, 5:305-14.

4. Bleyer A, O'Leary M, Barr R, Ries LAG, editors. Cancer Epidemiology in Older Adolescents and Young Adults 15 to 29 Years of Age, Including SEER Incidence and Survival: 1975-2000. National Cancer Institute, NIH Pub. No. 06-5767. Bethesda, MD 2006.

5. Bleyer A, Barr R, Hayes-Lattin B, Thomas D, Ellis C, Anderson B, et al. The distinctive biology of cancer in adolescents and young adults. Nat Rev Cancer. 2008;8:288-98.

6. Stiller CA, Trama A, Serraino D, Rossi S, Navarro C, Chirlaque MD, et al. Descriptive epidemiology of sarcomas in Europe: report from the RARECARE project. Eur J Cancer. 2013;49:684-95.

7. van der Graaf WT, Orbach D, Judson IR, Ferrari A. Soft tissue sarcomas in adolescents and young adults: a comparison with their paediatric and adult counterparts. Lancet Oncol. 2017;18:e166-75.

8. Honore C, Meeus P, Stoeckle E, Bonvalot S. Soft tissue sarcoma in France in 2015: Epidemiology, classification and organization of clinical care. J Visc Surg. 2015;152:223-30.

9. Fukushima T, Ogura K, Akiyama T, Takeshita K, Kawai A. Descriptive epidemiology and outcomes of bone sarcomas in adolescent and young adult patients in Japan. BMC Musculoskelet Disord. 2018;19:297.

10. Ogura K, Higashi T, Kawai A. Statistics of bone sarcoma in Japan: Report from the Bone and Soft Tissue Tumor Registry in Japan. J Orthop Sci. 2017;22:133-43.

11. Adams SH, Newacheck PW, Park MJ, Brindis CD, Irwin CEJ. Health insurance across vulnerable ages: patterns and disparities from adolescence to the early 30s. Pediatrics. 2007;119:e1033-9.

12. Keegan TH, Tao L, DeRouen MC, Wu XC, Prasad P, Lynch CF, et al. Medical care in adolescents and young adult cancer survivors: what are the biggest access-related barriers? J Cancer Surviv. 
2014;8:282-92.

13. Hsieh MC, Wu XC, Andrews PA, Chen VW. Racial and ethnic disparities in the incidence and trends of soft tissue sarcoma among adolescents and young adults in the United States, 1995-2008. J Adolesc Young Adult Oncol. 2013;2:89-94.

14. Herzog CE. Overview of sarcomas in the adolescent and young adult population. J Pediatr Hematol Oncol. 2005;27:215-8.

15. Ng VY, Scharschmidt TJ, Mayerson JL, Fisher JL. Incidence and survival in sarcoma in the United States: a focus on musculoskeletal lesions. Anticancer Res. 2013;33:2597-604.

16. Bergamaschi L, Bisogno G, Manzitti C, D'Angelo P, Milano GM, Scagnellato A, et al. Salvage rates and prognostic factors after relapse in children and adolescents with malignant peripheral nerve sheath tumors. Pediatr Blood Cancer. 2018;65.

17. Porter DE, Prasad V, Foster L, Dall GF, Birch R, Grimer RJ. Survival in malignant peripheral nerve sheath tumours: a comparison between sporadic and neurofibromatosis type 1-associated tumours. Sarcoma. 2009;2009:756395.

18. Valentin T, Le Cesne A, Ray-Coquard I, Italiano A, Decanter G, Bompas E, et al. Management and prognosis of malignant peripheral nerve sheath tumors: The experience of the French Sarcoma Group (GSF-GETO). Eur J Cancer. 2016, 56:77-84.

19. Evans DG, Baser ME, McGaughran J, Sharif S, Howard E, Moran A. Malignant peripheral nerve sheath tumours in neurofibromatosis 1. J Med Genet. 2002, 39:311-4.

20. Ingham S, Huson SM, Moran A, Wylie J, Leahy M, Evans DG. Malignant peripheral nerve sheath tumours in NF1: improved survival in women and in recent years. Eur J Cancer. 2011, 47:2723-8.

21. Quinn GP, Goncalves V, Sehovic I, Bowman ML, Reed DR. Quality of life in adolescent and young adult cancer patients: a systematic review of the literature. Patient Relat Outcome Meas. 2015;6:1951

\section{Tables}


Table 1. Patient characteristics by age group

\begin{tabular}{|c|c|c|c|c|c|c|c|c|c|c|c|}
\hline & \multirow{2}{*}{\multicolumn{2}{|c|}{$\begin{array}{l}\text { AYA } \\
\text { (15-39years) }\end{array}$}} & \multirow{2}{*}{\multicolumn{2}{|c|}{ Overall }} & \multirow{2}{*}{\multicolumn{2}{|c|}{$\begin{array}{l}\text { Child } \\
\text { ( } \leq 14 \text { years) }\end{array}$}} & \multirow{2}{*}{\multicolumn{2}{|c|}{$\begin{array}{l}\text { Adult } \\
\text { (40-64years) }\end{array}$}} & \multirow{2}{*}{\multicolumn{2}{|c|}{$\begin{array}{l}\text { Elderly } \\
\text { (65- years) }\end{array}$}} & \multirow[t]{3}{*}{$\mathrm{P}$ value } \\
\hline & & & & & & & & & & & \\
\hline & $\mathrm{N}$ & $\%$ & $\mathrm{~N}$ & $\%$ & $\mathrm{~N}$ & $\%$ & $\mathrm{~N}$ & $\%$ & $\mathrm{~N}$ & $\%$ & \\
\hline Total & 1467 & & 7759 & & 210 & & 2771 & & 3311 & & \\
\hline Sex & & & & & & & & & & & $<0.001$ \\
\hline Male & 778 & $53.0 \%$ & 4309 & $55.5 \%$ & 91 & $43.3 \%$ & 1571 & $56.7 \%$ & 1869 & $56.4 \%$ & \\
\hline Female & 689 & $47.0 \%$ & 3450 & $44.5 \%$ & 119 & $56.7 \%$ & 1200 & $43.3 \%$ & 1442 & $43.6 \%$ & \\
\hline Histologic grade & & & & & & & & & & & $<0.001$ \\
\hline Low & 301 & $20.5 \%$ & 1454 & $18.7 \%$ & 16 & $7.6 \%$ & 616 & $22.2 \%$ & 521 & $15.7 \%$ & \\
\hline High & 1166 & $79.5 \%$ & 6305 & $81.3 \%$ & 194 & $92.4 \%$ & 2155 & $77.8 \%$ & 2790 & $84.3 \%$ & \\
\hline Histologic subtype & & & & & & & & & & & $<0.001$ \\
\hline MRLS & 286 & $19.5 \%$ & 956 & $12.3 \%$ & 3 & $1.4 \%$ & 449 & $16.2 \%$ & 218 & $6.6 \%$ & \\
\hline SySa & 259 & $17.7 \%$ & 555 & $7.2 \%$ & 36 & $17.1 \%$ & 188 & $6.8 \%$ & 72 & $2.2 \%$ & \\
\hline MPNST & 130 & $8.9 \%$ & 478 & $6.2 \%$ & 14 & $6.7 \%$ & 173 & $6.2 \%$ & 161 & $4.9 \%$ & \\
\hline PNET & 111 & $7.6 \%$ & 210 & $2.7 \%$ & 29 & $13.8 \%$ & 54 & $1.9 \%$ & 16 & $0.5 \%$ & \\
\hline RMS & 94 & $6.4 \%$ & 271 & $3.5 \%$ & 79 & $37.6 \%$ & 51 & $1.8 \%$ & 47 & $1.4 \%$ & \\
\hline UPS & 90 & $6.1 \%$ & 2030 & $26.2 \%$ & 3 & $1.4 \%$ & 629 & $22.7 \%$ & 1308 & $39.5 \%$ & \\
\hline ASPS & 77 & $5.2 \%$ & 110 & $1.4 \%$ & 13 & $6.2 \%$ & 17 & $0.6 \%$ & 3 & $0.1 \%$ & \\
\hline EpiSa & 73 & $5.0 \%$ & 146 & $1.9 \%$ & 4 & $1.9 \%$ & 51 & $1.8 \%$ & 19 & $0.5 \%$ & \\
\hline $\operatorname{CCS}$ & 51 & $3.5 \%$ & 107 & $1.4 \%$ & 2 & $1.0 \%$ & 37 & $1.3 \%$ & 17 & $0.5 \%$ & \\
\hline High grade others & 182 & $12.4 \%$ & 2109 & $27.2 \%$ & 16 & $7.6 \%$ & 790 & $28.5 \%$ & 1121 & $33.9 \%$ & \\
\hline Low grade others & 114 & $7.8 \%$ & 787 & $10.1 \%$ & 11 & $5.2 \%$ & 332 & $12.0 \%$ & 330 & $10.0 \%$ & \\
\hline Tumor size $(\mathrm{cm})$ & & & & & & & & & & & $<0.001$ \\
\hline$\leq 5 \mathrm{~cm}$ & 484 & $33.0 \%$ & 2142 & $27.6 \%$ & 97 & $46.2 \%$ & 716 & $25.8 \%$ & 845 & $25.5 \%$ & \\
\hline$>5 \mathrm{~cm}$ and $\leq 10 \mathrm{~cm}$ & 530 & $36.1 \%$ & 2916 & $37.6 \%$ & 78 & $37.1 \%$ & 989 & $35.7 \%$ & 1319 & $39.8 \%$ & \\
\hline$>10 \mathrm{~cm}$ & 333 & $22.7 \%$ & 2138 & $27.6 \%$ & 21 & $10.0 \%$ & 839 & $30.3 \%$ & 945 & $28.5 \%$ & \\
\hline Unknown & 120 & $8.2 \%$ & 563 & $7.3 \%$ & 14 & $6.7 \%$ & 167 & $6.0 \%$ & 202 & $6.1 \%$ & \\
\hline Tumor location & & & & & & & & & & & $<0.001$ \\
\hline
\end{tabular}




\begin{tabular}{|c|c|c|c|c|c|c|c|c|c|c|c|}
\hline Upper extremity & 182 & $12.4 \%$ & 963 & $12.4 \%$ & 48 & $22.9 \%$ & 289 & $10.4 \%$ & 444 & $13.4 \%$ & \\
\hline Lower extremity & 671 & $45.7 \%$ & 3904 & $50.3 \%$ & 88 & $41.9 \%$ & 1412 & $51.0 \%$ & 1733 & $52.3 \%$ & \\
\hline Trunk & 486 & $33.1 \%$ & 2493 & $32.1 \%$ & 45 & $21.4 \%$ & 950 & $34.3 \%$ & 1012 & $30.6 \%$ & \\
\hline Head and neck & 80 & $5.5 \%$ & 216 & $2.8 \%$ & 17 & $8.1 \%$ & 56 & $2.0 \%$ & 63 & $1.9 \%$ & \\
\hline Multiple disease & 48 & $3.3 \%$ & 183 & $2.4 \%$ & 12 & $5.7 \%$ & 64 & $2.3 \%$ & 59 & $1.8 \%$ & \\
\hline Surgery & 1120 & $76.3 \%$ & 6200 & $79.9 \%$ & 150 & $71.4 \%$ & 2227 & $80.4 \%$ & 2703 & $81.6 \%$ & $<0.001$ \\
\hline Chemotherapy & 795 & $54.6 \%$ & 2567 & $33.2 \%$ & 148 & $72.2 \%$ & 1159 & $41.9 \%$ & 465 & $14.1 \%$ & $<0.001$ \\
\hline Adjuvant & 536 & $67.4 \%$ & 1653 & $64.4 \%$ & 112 & $75.5 \%$ & 760 & $65.6 \%$ & 245 & $52.7 \%$ & \\
\hline Palliative & 246 & $30.9 \%$ & 858 & $33.4 \%$ & 29 & $19.6 \%$ & 371 & $32.0 \%$ & 212 & $45.6 \%$ & \\
\hline Unknown & 13 & $1.6 \%$ & 56 & $2.2 \%$ & 7 & $4.7 \%$ & 28 & $2.4 \%$ & 8 & $1.7 \%$ & \\
\hline Radiotherapy & 370 & $25.5 \%$ & 1925 & $25.0 \%$ & 80 & $39.6 \%$ & 645 & $23.4 \%$ & 830 & $25.2 \%$ & $<0.001$ \\
\hline Adjuvant & 196 & $53.0 \%$ & 1156 & $60.1 \%$ & 46 & $57.5 \%$ & 398 & $61.7 \%$ & 516 & $62.2 \%$ & \\
\hline Palliative & 70 & $18.9 \%$ & 322 & $16.7 \%$ & 4 & $5.0 \%$ & 114 & $17.7 \%$ & 134 & $16.1 \%$ & \\
\hline Radical & 94 & $25.4 \%$ & 393 & $20.4 \%$ & 30 & $37.5 \%$ & 108 & $16.7 \%$ & 161 & $19.4 \%$ & \\
\hline Unknown & 10 & $2.7 \%$ & 54 & $2.8 \%$ & 0 & $0 \%$ & 25 & $3.9 \%$ & 19 & $2.3 \%$ & \\
\hline
\end{tabular}

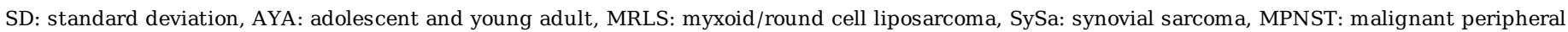

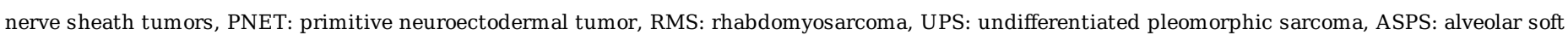
part sarcoma, EpiSa: epithelioid sarcoma, CCS: clear-cell sarcoma 
Table 2. Univariate and multivariate analyses of prognostic factors for overall cancer survival in soft tissue sarcoma

\begin{tabular}{|c|c|c|c|c|c|c|}
\hline & \multirow[t]{2}{*}{ No. of patients (\%) } & \multirow[t]{2}{*}{ 5-year survival (\%) } & \multicolumn{2}{|c|}{ Univariate analysis } & \multicolumn{2}{|c|}{ Multivariate analysis } \\
\hline & & & Hazard ratio $(95 \% \mathrm{CI})$ & $P$ value & Hazard ratio $(95 \% \mathrm{CI})$ & $P$ value \\
\hline Total & 5853 & $72.9 \%$ & & & & \\
\hline \multicolumn{7}{|l|}{ Age } \\
\hline AYA (15-39 years) & 1149 & $69.9 \%$ & Reference & & Reference & \\
\hline Child ( $\leq 14$ years) & 165 & $71.8 \%$ & $0.80(0.55-1.17)$ & 0.25 & $0.94(0.59-1.49)$ & 0.800 \\
\hline Adult (40-64 years) & 2127 & $75.2 \%$ & $0.84(0.71-0.99)$ & 0.033 & $1.35(1.11-1.65)$ & 0.003 \\
\hline Elderly ( $\geq 65$ years) & 2412 & $72.5 \%$ & $0.98(0.84-1.15)$ & 0.812 & $2.28(1.85-2.79)$ & $<0.001$ \\
\hline \multicolumn{7}{|l|}{ Sex } \\
\hline Female & 2638 & $75.5 \%$ & Reference & & Reference & \\
\hline Male & 3215 & $70.7 \%$ & $1.33(1.18-1.50)$ & $<0.001$ & $1.14(0.99-1.31)$ & 0.061 \\
\hline \multicolumn{7}{|l|}{ Histologic grade } \\
\hline Low & 1065 & $93.2 \%$ & Reference & & Reference & \\
\hline High & 4788 & $68.2 \%$ & $6.96(5.05-9.58)$ & $<0.001$ & $3.41(2.37-4.91)$ & $<0.001$ \\
\hline \multicolumn{7}{|l|}{ Tumor size(cm) } \\
\hline$\leq 5 \mathrm{~cm}$ & 1635 & $85.8 \%$ & Reference & & Reference & \\
\hline$>5 \mathrm{~cm}$ and $\leq 10 \mathrm{~cm}$ & 2216 & $74.3 \%$ & $2.23(1.84-2.70)$ & $<0.001$ & $1.71(1.38-2.12)$ & $<0.001$ \\
\hline$>10 \mathrm{~cm}$ & 1587 & $57.2 \%$ & $4.07(3.38-4.90)$ & $<0.001$ & $2.57(2.06-3.19)$ & $<0.001$ \\
\hline \multicolumn{7}{|l|}{ Tumor location } \\
\hline Upper extremity & 728 & $83.8 \%$ & Reference & & Reference & \\
\hline Lower extremity & 2955 & $76.8 \%$ & $1.38(1.09-1.75)$ & 0.007 & $1.14(0.88-1.49)$ & 0.331 \\
\hline Trunk & 1870 & $65.7 \%$ & $2.51(1.98-3.17)$ & $<0.001$ & $1.77(1.34-2.32)$ & $<0.001$ \\
\hline Head and neck & 173 & $66.2 \%$ & $2.62(1.83-3.75)$ & $<0.001$ & $1.73(1.07-2.78)$ & 0.025 \\
\hline Multiple & 127 & $35.2 \%$ & $6.69(4.87-9.20)$ & $<0.001$ & $2.32(1.59-3.39)$ & $<0.001$ \\
\hline \multicolumn{7}{|l|}{ Surgery } \\
\hline- & 692 & $51.7 \%$ & Reference & & Reference & \\
\hline+ & 4272 & $77.3 \%$ & $0.315(0.278-0.357)$ & $<0.001$ & $0.58(0.49-0.69)$ & $<0.001$ \\
\hline \multicolumn{7}{|l|}{ Limb salvage status } \\
\hline Limb salvage & 5072 & $76.4 \%$ & Reference & & Reference & \\
\hline
\end{tabular}


Chemotherapy

\begin{tabular}{|c|c|c|c|c|c|c|}
\hline- & 3737 & $83.1 \%$ & Reference & & Reference & \\
\hline+ & 2106 & $56.4 \%$ & $2.69(2.39-3.03)$ & $<0.001$ & 1.59 (1.35-1.87) & $<0.001$ \\
\hline \multicolumn{7}{|l|}{ Radiation } \\
\hline- & 4231 & $79.6 \%$ & Reference & & & \\
\hline+ & 1604 & $56.7 \%$ & $2.42(2.15-2.72)$ & $<0.001$ & $1.38(1.20-1.59)$ & $<0.001$ \\
\hline \multicolumn{7}{|l|}{ Metastasis } \\
\hline- & 5030 & $80.5 \%$ & Reference & & Reference & \\
\hline ] & 771 & $22.9 \%$ & $7.95(7.04-8.98)$ & $<0.001$ & $4.68(3.97-5.51)$ & $<0.001$ \\
\hline \multicolumn{7}{|l|}{ Tumor Depth } \\
\hline Superficial & 1453 & $87.3 \%$ & Reference & & Reference & \\
\hline Deep to fascia & 4280 & $78.3 \%$ & $2.83(2.35-3.42)$ & $<0.001$ & $1.34(1.07-1.67)$ & 0.010 \\
\hline
\end{tabular}

AYA: Adolescent and Young Adult, CI: confidence interval 
Table 3. Characteristics of MPNST patients according to age group

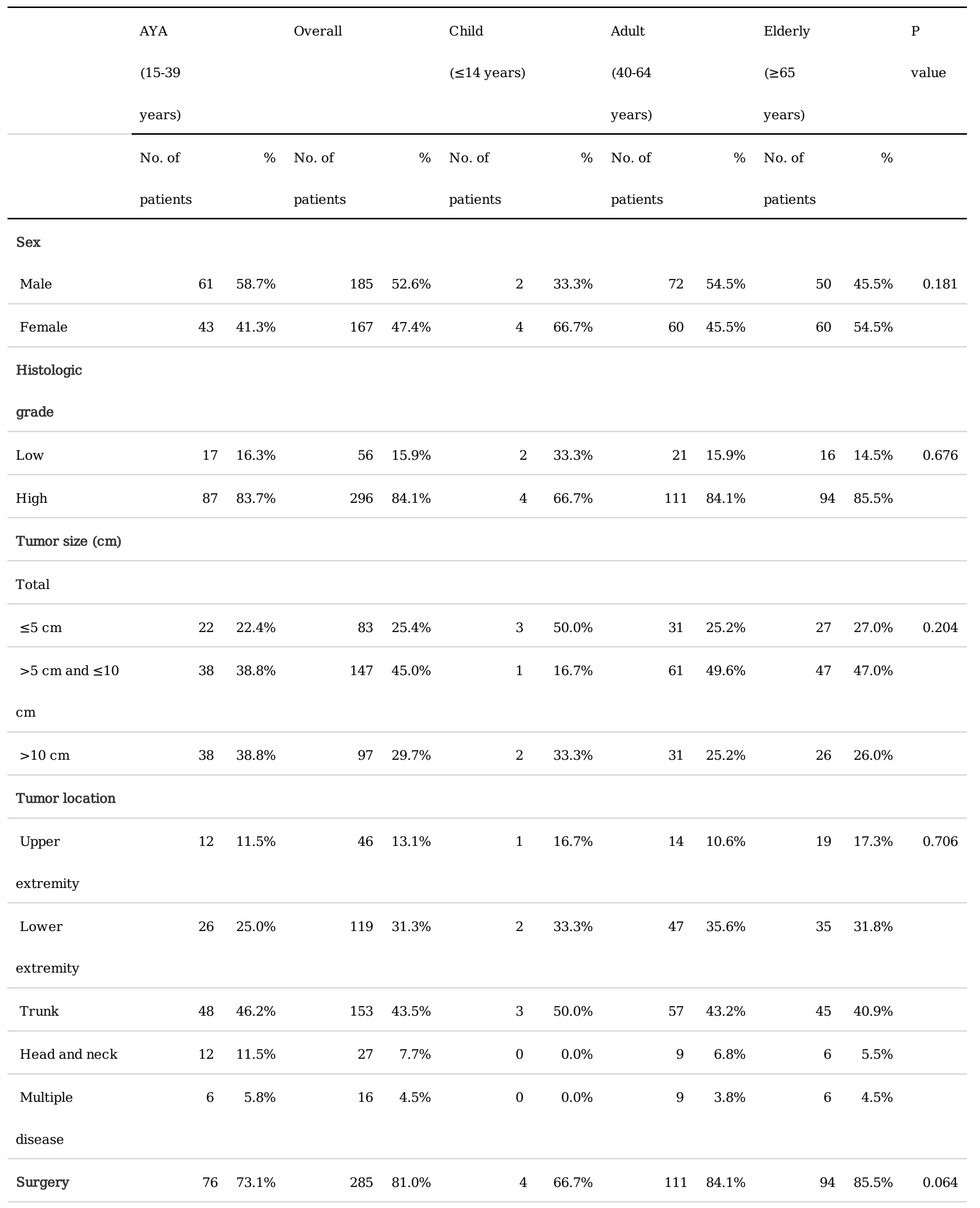




\begin{tabular}{|c|c|c|c|c|c|c|c|c|c|c|c|}
\hline Chemotherapy & 57 & $55.3 \%$ & 134 & $38.2 \%$ & 2 & $33.3 \%$ & 63 & $47.7 \%$ & 12 & $10.9 \%$ & \\
\hline Adjuvant & 28 & $49.1 \%$ & 64 & $47.8 \%$ & 2 & $100 \%$ & 30 & $47.6 \%$ & 4 & $33.3 \%$ & $<0.001$ \\
\hline Palliative & 29 & $50.9 \%$ & 67 & $50.0 \%$ & 0 & $0 \%$ & 30 & $47.6 \%$ & 8 & $66.7 \%$ & \\
\hline Unknown & 0 & $0 \%$ & 3 & $2.2 \%$ & 0 & $0 \%$ & 3 & $4.8 \%$ & 0 & $0 \%$ & \\
\hline Radiotherapy & 34 & $33.0 \%$ & 106 & $30.2 \%$ & 1 & $16.7 \%$ & 42 & $31.8 \%$ & 29 & $26.4 \%$ & \\
\hline Adjuvant & 14 & $41.2 \%$ & 56 & $52.8 \%$ & 1 & $100 \%$ & 23 & $54.8 \%$ & 18 & $62.1 \%$ & 0.619 \\
\hline Palliative & 13 & $38.2 \%$ & 28 & $26.4 \%$ & 0 & $0 \%$ & 11 & $26.2 \%$ & 4 & $13.8 \%$ & \\
\hline Radical & 6 & $17.6 \%$ & 20 & $18.9 \%$ & 0 & $0 \%$ & 7 & $16.7 \%$ & 7 & $24.1 \%$ & \\
\hline Unknown & 1 & $2.9 \%$ & 2 & $1.9 \%$ & 0 & $0 \%$ & 0 & $0 \%$ & 0 & $0 \%$ & \\
\hline \multirow[t]{2}{*}{ Metastasis } & 21 & $20.2 \%$ & 51 & $14.7 \%$ & 0 & $0.0 \%$ & 18 & $13.7 \%$ & 12 & $11.2 \%$ & \\
\hline & & & & & & & & & & & 0.197 \\
\hline \multicolumn{12}{|l|}{ Depth } \\
\hline superficial & 16 & $16.0 \%$ & 87 & $25.7 \%$ & 0 & $0.0 \%$ & 32 & $25.0 \%$ & 39 & $37.1 \%$ & 0.003 \\
\hline deep & 84 & $84.0 \%$ & 252 & $74.3 \%$ & 6 & $100.0 \%$ & 96 & $75.0 \%$ & 66 & $62.9 \%$ & \\
\hline
\end{tabular}

MPNST: malignant peripheral nerve sheath tumors, CI: confidence interval 
Table 4. Univariate and multivariate analyses of prognostic factors for cancer survival in MPNST patients

\begin{tabular}{|c|c|c|c|c|c|}
\hline & \multicolumn{3}{|c|}{ Univariate analysis } & \multicolumn{2}{|c|}{ Multivariate analysis } \\
\hline & No. of patients (\%) & Hazard ratio $(95 \%$ CI) & $\mathrm{P}$ value & Hazard ratio $(95 \% \mathrm{CI})$ & $P$ value \\
\hline Total & 256 & & & & \\
\hline \multicolumn{6}{|l|}{ Age } \\
\hline AYA (15-39 years) & 68 & Reference & & Reference & \\
\hline Child ( $\leq 14$ years) & 4 & $0.000(0.000-2.69 \mathrm{E}+$ (स) & 0.95 & $0.000(0.000-)$ & 0.971 \\
\hline Adult (40-59 years) & 101 & $0.62(0.39-0.97)$ & 0.036 & $0.73(0.43-1.25)$ & 0.250 \\
\hline Elderly ( $\geq 65$ years) & 83 & $0.45(0.26-0.77)$ & 0.004 & $0.71(0.36-1.40)$ & 0.318 \\
\hline \multicolumn{6}{|l|}{ Sex } \\
\hline Female & 122 & Reference & & Reference & \\
\hline Male & 134 & $1.63(1.07-2.46)$ & 0.022 & $1.17(0.73-1.89)$ & 0.510 \\
\hline \multicolumn{6}{|l|}{ Histologic grade } \\
\hline Low & & Reference & & Reference & \\
\hline High & & 4.09 (1.66-10.06) & 0.002 & $2.71(0.94-7.84)$ & 0.065 \\
\hline \multicolumn{6}{|l|}{ Tumor size } \\
\hline$\leq 5 \mathrm{~cm}$ & 66 & Reference & & Reference & \\
\hline$>5 \mathrm{~cm}$ and $\leq 10 \mathrm{~cm}$ & 125 & $2.65(1.28-5.46)$ & 0.009 & $2.20(1.01-4.77)$ & 0.046 \\
\hline$>10 \mathrm{~cm}$ & 65 & $3.71(1.78-7.72)$ & $<0.001$ & $2.54(1.17-5.50)$ & 0.018 \\
\hline \multicolumn{6}{|l|}{ Tumor location } \\
\hline Upper extremity & 34 & Reference & & Reference & \\
\hline Lower extremity & 89 & $1.00(0.49-2.01)$ & 0.99 & $0.74(0.34-1.58)$ & 0.430 \\
\hline Trunk & 110 & $1.10(0.57-2.14)$ & 0.775 & $0.78(0.38-1.61)$ & 0.504 \\
\hline Head and neck & 16 & $1.53(0.64-3.70)$ & 0.342 & $1.14(0.41-3.20)$ & 0.801 \\
\hline Multiple & 7 & $2.59(1.00-6.70)$ & 0.049 & $1.48(0.51-4.31)$ & 0.474 \\
\hline \multicolumn{6}{|l|}{ Surgery } \\
\hline- & 67 & Reference & & Reference & \\
\hline+ & 285 & $0.28(0.18-0.42)$ & $<0.001$ & $0.51(0.29-0.92)$ & 0.024 \\
\hline
\end{tabular}




\begin{tabular}{cccccc}
-217 & Reference & \multicolumn{3}{c}{ Reference } \\
\hline+ & 134 & $2.44(1.62-3.67)$ & $<0.001$ & $1.17(0.67-2.06)$ & 0.579 \\
\hline
\end{tabular}

\section{Radiation}

\begin{tabular}{|c|c|c|c|c|c|}
\hline- & 245 & Reference & & Reference & \\
\hline+ & 106 & $2.44(1.63-3.65)$ & $<0.001$ & $1.70(0.67-2.06)$ & 0.035 \\
\hline \multicolumn{6}{|l|}{ Metastasis } \\
\hline- & 235 & Reference & & Reference & \\
\hline ૫ & 21 & $5.47(3.57-8.37)$ & $<0.001$ & $3.11(1.76-5.51)$ & $<0.001$ \\
\hline \multicolumn{6}{|l|}{ Tumor Depth } \\
\hline Superficial & 68 & Reference & & Reference & \\
\hline Deep to fascia & 125 & $3.92(1.89-8.10)$ & $<0.001$ & $2.71(1.23-5.98)$ & 0.014 \\
\hline
\end{tabular}

AYA: Adolescent and Young Adult, CI: confidence interval

\section{Figures}


a Overall

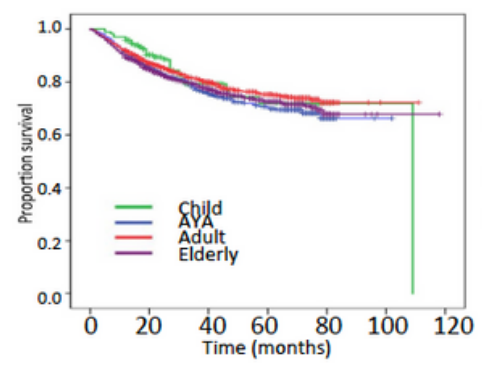

e ASPS

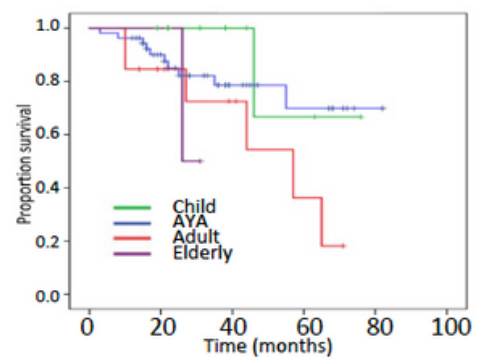

e RMS

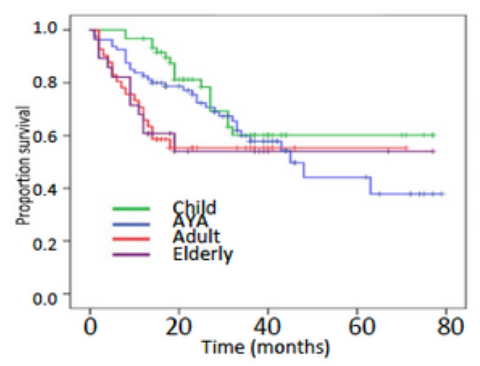

b UPS

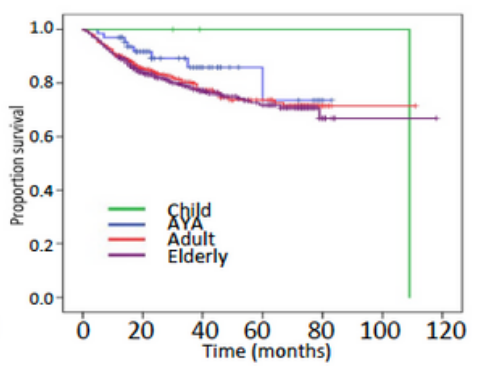

f CCS

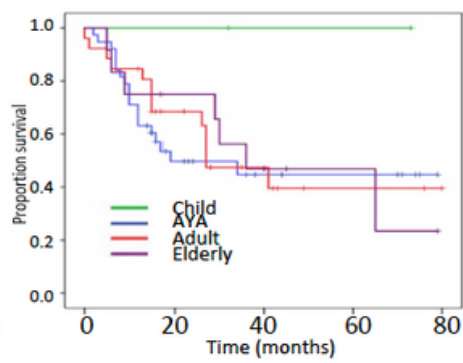

SySa

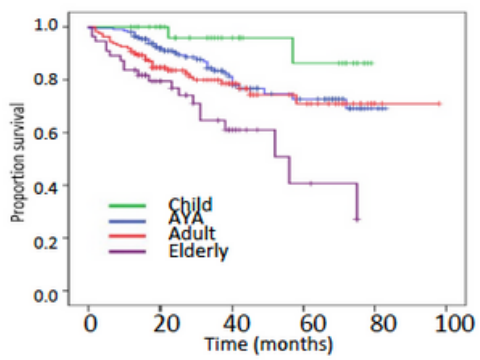

c MRLS
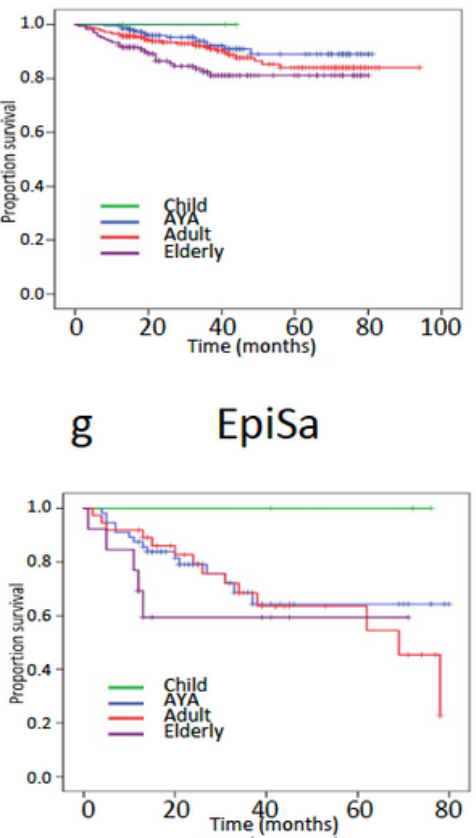

k High grade others g EpiSa

d $\quad$ MPNST

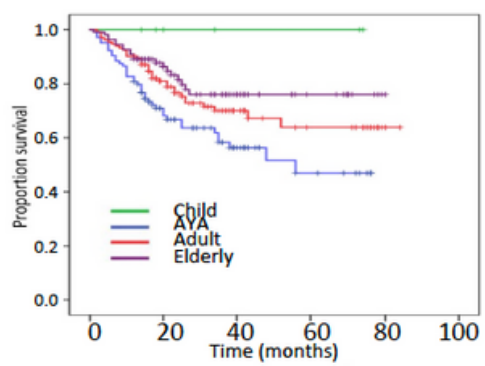

h PNET

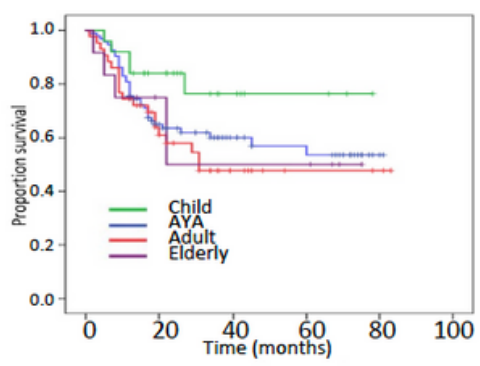

I Low grade others

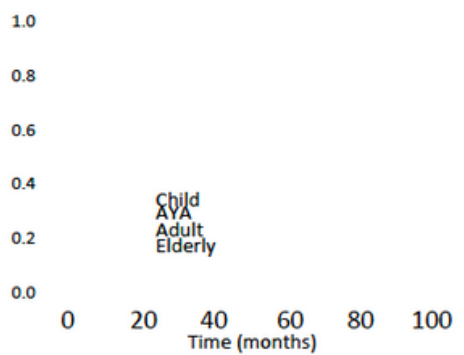

Figure 1

Kaplan-Meier survival curves showing survival rates for all tumors. The results show survival for overall sarcomas (a), UPS (b), MRLS (c), MPNST (d), ASPS (e), CCS (f), EpiSa (g), PNET (h), RMS (i), SySa (j), other high-grade tumors $(\mathrm{k})$, and other low-grade tumors (l) stratified by age. Child: $\leq 14$ years, adolescent and young adult (AYA): 15-39 years, adult: $40-64$ years, and elderly: $\geq 65$ years. 